Artículo

\title{
Compuestos fenólicos y capacidad antirradicalaria de cinco accesiones silvestres de Portulaca oleracea L. obtenidas con tres solventes
}

\author{
José Nabor Martínez-López ${ }^{1 \S}$ \\ Jorge Ariel Torres-Castillo ${ }^{1}$ \\ Guadalupe Concepción Rodríguez-Castillejos ${ }^{2}$ \\ José Guadalupe Martínez-Ávalos ${ }^{1}$ \\ Emmanuel Ortíz-Espinoza ${ }^{3}$ \\ Alicia Guadalupe Marroquín-Cardona ${ }^{4}$
}

${ }^{1}$ Universidad Autónoma de Tamaulipas-Instituto de Ecología Aplicada. Av. División del Golfo 356, Colonia Libertad, Ciudad Victoria, Tamaulipas, México. CP. 87019. (jorgearieltorres@hotmail.com, jmartin@uat.edu.mx). ${ }^{2}$ Universidad Autónoma de Tamaulipas-Unidad Académica Multidisciplinaria Reynosa Aztlán. Calle 16 y Lago de Chapala, Col. Aztlán, Ciudad Reynosa, Tamaulipas, México. CP. 88740. (gcastillejos@ docentes.uat.edu.mx). ${ }^{3}$ Universidad Potosina, SC. Ignacio Comonfort \#805, Zona Centro, San Luis Potosí, México. CP. 78000. (space2_em@ hotmail.com). ${ }^{4}$ Universidad Autónoma de Nuevo León-Facultad de Medicina Veterinaria y Zootecnia. Francisco Villa s/n, Ex Hacienda El Canadá, General Escobedo, Nuevo León, México. CP. 66050. (alicia.marroquincr@uanl.edu.mx).

§Autor para correspondencia: nabor_marlo89@hotmail.com.

\section{Resumen}

La verdolaga (Portulaca oleracea L.) es reconocida por sus altos niveles de compuestos bioactivos con propiedades antioxidantes. El objetivo del trabajo fue determinar la variación de metabolitos fenólicos y sus capacidades antirradicalarias en accesiones silvestres de $P$. oleracea. La investigación se realizó con muestras de cinco municipios de Tamaulipas, recolectadas en octubre 2018. Se determinaron los contenidos de compuestos fenólicos totales (CFT) y capacidad colectora de radicales libres contra ABTS (ácido 2,2'-azino-bis- (3-etilbenzotiazolina-6-sulfónico)) y DPPH (2,2-difenil-1-picrilhidracilo). Las extracciones se realizaron de partes vegetativas usando tres solventes: acetona, agua y etanol. La accesión de las localidades de Abasolo y Padilla fueron la que presentaron mayor cantidad de CFT con $5.8938 \pm 0.03$ y $5.3742 \pm 0.11 \mathrm{mEAG} \mathrm{g}^{-1}$ PS, respectivamente, usando agua en la extracción. Respecto a la capacidad colectora de radicales libres contra ABTS la accesión de Abasolo y Jiménez registraron los valores más altos, con 3.27 \pm 0.06 y $3.2226 \pm 0.04 \mathrm{mM}$ ET g ${ }^{-1}$ PS. En cuanto a nivel contra radicales DPPH, la accesión de Abasolo fue la más alta con $2.02040 .05 \mathrm{mM} \mathrm{ET} \mathrm{g}^{-1}$ PS usando agua en la extracción. El agua fue el mejor solvente para la extracción de los contenidos determinados. Se observó una heterogeneidad en la composición y niveles de los parámetros evaluados entre las accesiones. Las accesiones silvestres de $P$. oleracea representan reservorios de compuestos fenólicos y capacidad colectora de radicales libres, incluyendo las variedades cultivadas y silvestres.

Palabras clave: antioxidantes, planta comestible, variación fitoquímicos.

Recibido: junio de 2021

Aceptado: agosto de 2021 


\section{Introducción}

Los productos vegetales con contenidos de antioxidantes se consideran eficientes para reducir el estrés oxidativo, por lo cual, son consumidos para reducir dicho proceso en las células. La caracterización de la actividad antioxidante de las plantas comestibles es la base para poder relacionarlas con funciones biológicas potenciales, efectos benéficos y para programas de mejoramiento y aprovechamiento de cultivos (Top et al., 2014; Urbizu-González et al., 2017).

Los compuestos fenólicos son considerados como antioxidantes y se han reportado en muchas especies de frutas, hortalizas y cereales consumidos como parte de la dieta humana. De forma endógena son los principales responsables de funciones fisiológicas y ecológicas y se ha observado que sus concentraciones varían frente a situaciones de estrés biótico y abiótico (Frontela et al., 2010; Sauceda et al., 2011). Por esto, es importante reconocer la dinámica de estos compuestos, asociada al estrés y a la variación ambiental, ya que esta información, puede servir de indicador para desarrollar estrategias de manejo para su mejor aprovechamiento (Qasim et al., 2017).

Sobre todo, en el caso de especies de interés alimentario y especias, ya que las variaciones en los contenidos de compuestos fenólicos y sus actividades antioxidantes y antirradicalarias, pueden considerarse como parámetros indicativos de calidad y beneficios potenciales a través de su consumo (Capecka et al., 2005; Ninfali et al., 2005; Cobaleda-Velasco et al., 2017).

Recientemente, se ha señalado que Portulaca oleracea L. es una buena fuente de compuestos benéficos para la salud humana, incluyendo ácidos grasos omega-3 y $\beta$-caroteno, vitaminas, aminoácidos esenciales, $\alpha$-tocofenoles, mucílagos, compuestos antioxidantes como glutatión y ácido ascórbico, compuestos fenólicos y minerales (Oliveira et al., 2009; Ramadan et al., 2017; Khodadadi et al., 2018; Allahmoradi et al., 2018). Esta planta es considerada como una maleza de distribución casi mundial, aunque en muchas regiones es apreciada por sus propiedades nutrimentales y medicinales.

Recientemente, el potencial antioxidante de $P$. oleracea se ha considerado como un criterio para su consumo y aprovechamiento, por lo que la exploración de accesiones que tengan niveles considerables de estos compuestos representa una oportunidad para estudiar las variaciones de estos compuestos en planes de mejoramiento (Alu'datt et al., 2019; Habibian et al., 2020). En México, esta planta se consume de diversas maneras y se ha remarcado su amplia distribución y plasticidad para adaptarse a diversos ambientes (Gutiérrez et al., 2007; Alam et al., 2014; Román et al., 2018).

Respecto a la acumulación de componentes antioxidantes, se ha observado que muchas plantas varían su composición fitoquímica dependiendo de las condiciones ambientales y de crecimiento (Bautista et al., 2016; Caverzan et al., 2016), lo que puede comprometer su calidad para fines alimentarios o medicinales (Bartolini et al., 2018). Dichas fluctuaciones se han observado en variedades cultivadas y silvestres de $P$. oleracea, donde se destaca que las variedades cultivadas presentan mayor contenido de fitoquímicos y actividades antioxidantes (Nemzer et al., 2020; Sdouga et al., 2020). 
No obstante, hay que considerar que las formas silvestres de algunas especies pueden ser reservorios de compuestos diversos en proporciones diferentes a las cultivadas debido a la influencia de las condiciones ambientales y las características genéticas (Mocan et al., 2017). En la zona noreste de México, el consumo y cultivo de la $P$. oleracea es bastante limitado, aún y cuando es una planta de amplia distribución, asociada a los asentamientos y zonas agrícolas. Esta planta representa un recurso alimenticio que no se explota y que además, ha demostrado tener el potencial para suplementar la alimentación con compuestos antioxidantes.

En este sentido se ha sugerido la exploración nutrimental y nutracéutica de las poblaciones de esta especie para determinar sus respuestas a las condiciones ambientales para desarrollar estrategias que permitan la selección y aprovechamiento de este recurso natural.

Por lo tanto, el objetivo de esta investigación fue determinar la variación de los contenidos de compuestos fenólicos y antioxidantes en accesiones de $P$. oleracea crecidas en cinco municipios de la parte central de Tamaulipas donde se localizaron poblaciones creciendo en forma no cultivada, con el fin de contribuir al conocimiento del material vegetal de la región y sugerir su aprovechamiento.

\section{Materiales y métodos}

\section{Recolecta de material vegetal}

El material vegetal de $P$. oleracea fue recolectado en los municipios de Abasolo, Jiménez, Llera, Padilla y San Nicolás, Tamaulipas, México (Cuadro 1). La especie fue identificada en el herbario de la Universidad Autónoma de Tamaulipas. Los sitios fueron seleccionados por la presencia de la planta, misma que no se había reportado con anterioridad, además la actividad agrícola en esas zonas se realiza con poca aplicación de agroquímicos.

Cuadro 1. Ubicación geográfica de cada accesión de $P$. oleracea en la zona 14 dada en UTM, el tipo clima dado por la clasificación Koppen (García, 2004).

\begin{tabular}{cccc}
\hline Accesión & Coordenadas & Clima & Municipio \\
\hline AB & $2660772.51 \mathrm{~N}, 563071.21 \mathrm{E}$ & BSh & Abasolo \\
JN & $2677473.20 \mathrm{~N}, 52204.40 \mathrm{E}$ & BSh & Jiménez \\
LL & $2578673.3 \mathrm{~N}, 497649.70 \mathrm{E}$ & BSh & Llera \\
PA & $2687973.20 \mathrm{~N}, 50208.40 \mathrm{O}$ & Cfa & Padilla \\
SN & $2731097.59 \mathrm{~N}, 517130.63 \mathrm{E}$ & BSk & San Nicolás \\
\hline
\end{tabular}

\section{Preparación de extractos}

El material recolectado se lavó con agua corriente para eliminar partículas de suelo y restos vegetales, posteriormente, se secó a $50{ }^{\circ} \mathrm{C}$ por $96 \mathrm{~h}$ en un horno de aire forzado. El material seco se pulverizó con un molino eléctrico y se tamizó a través de una malla de $700 \mu \mathrm{m}$ (US Standard sieve). El material vegetal fue sometido a extracción con tres solventes, para la extracción con etanol y acetona se usó una proporción 1:4 (p/v) y para la extracción con agua destilada se usó una proporción 1:6 (p/v). 
La mezcla de tejido y solventes se agitó por 20 min y posteriormente se centrifugó a 8000 x g por 10 min. El sobrenadante se recuperó y se usó como fuente de compuestos fenólicos para determinaciones posteriores. Los extractos se almacenaron a $-20{ }^{\circ} \mathrm{C}$ hasta su uso.

\section{Detección de compuestos fenólicos totales (CFT)}

Los compuestos fenólicos totales se cuantificaron con el reactivo de Folin-Ciocalteu (Singleton et al., 1999), usando una curva estándar preparada con ácido gálico (Sigma-Aldrich, St. Louis, MO, USA) con un rango de 100 a $500 \mu \mathrm{g} \mathrm{ml}^{-1}$. Las reacciones se prepararon mezclando las muestras con $250 \mu \mathrm{l}$ del reactivo de Folin-Ciocalteu (Sigma-Aldrich, St. Louis, MO, USA) y $750 \mu 1$ de $\mathrm{Na}_{2} \mathrm{CO}_{3}$ al $20 \%$, después se incubaron en oscuridad por $2 \mathrm{~h}$ a $37^{\circ} \mathrm{C}$. Posteriormente la absorbancia se registró a $765 \mathrm{~nm}$. Los resultados se reportaron en miligramos equivalentes de ácido gálico por gramo de tejido en peso seco ( $\left.\mathrm{mEAG} \mathrm{g} \mathrm{g}^{-1} \mathrm{PS}\right)$.

\section{Detección de capacidad colectora contra el radical libre DPPH}

Para la inhibición del radical libre DPPH (2,2 diphenyl-1-pricrylhydrazyl) se utilizó el método reportado por Brand-Williams et al. (1995), $50 \mu \mathrm{l}$ de los extractos se mezclaron con $2.95 \mathrm{ml}$ del reactivo DPPH (Sigma-Aldrich, St. Louis, MO, USA) al $60 \mu \mathrm{M}$ y se dejaron reaccionar por 30 min, de forma simultánea, se preparó una curva estándar con Trolox (Sigma-Aldrich, St. Louis, MO, USA) en un rango de 100 a $1200 \mu \mathrm{M}$ y se hicieron reaccionar en la misma forma que las muestras con el DPPH. Posterior a la incubación, todas las muestras fueron leídas a $517 \mathrm{~nm}$ y la absorbancia se usó para calcular la concentración de acuerdo con la curva estándar. Los resultados se expresaron como $\mathrm{mM}$ equivalentes de Trolox por gramo de peso seco ( $\mathrm{mM} \mathrm{ET} \mathrm{g}^{-1} \mathrm{PS}$ ).

\section{Detección de capacidad colectora contra el radical libre ABTS}

La capacidad colectora contra el radical libre ABTS (2,2'-azino-bis (3-ethylbenzothiazoline-6sulfonic acid) fue evaluada de acuerdo con el método reportado por Re et al., (1999). Una solución concentrada se preparó con $7 \mathrm{mM}$ de ABTS (Sigma-Aldrich, St. Louis, MO, USA) y $2.45 \mathrm{mM}$ de persulfato de potasio (Sigma-Aldrich, St. Louis, MO, USA), la cual se dejó reposar por $12 \mathrm{~h}$ en la oscuridad para activarla. Posteriormente, esta solución se ajustó con etanol hasta alcanzar una absorbancia de 0.7 0a $734 \mathrm{~nm}$. Los extractos en presencia del reactivo ABTS se incubaron por 6 min en la oscuridad y posteriormente se registró su absorbancia, esta se comparó con una curva estándar con Trolox (Sigma-Aldrich, St. Louis, MO, USA) en un rango de concentraciones de 100 a $1200 \mu \mathrm{M}$. La capacidad colectora contra ABTS se calculó y expresó como mM ET g ${ }^{-1}$ PS.

\section{Análisis estadístico}

Se realizaron tres extracciones con cada solvente para cada accesión y a todas las extracciones se les realizaron las determinaciones por triplicado. Para normalizar los datos, todos fueron transformados con la función box-cox en el programa estadístico PAST (Hammer et al., 2001). Se realizó un análisis de varianza seguido de una comparación de medias con la prueba de Tukey $(\alpha=$ $0.05)$ en aquellos tratamientos donde se encontraron diferencias estadísticamente significativas. 


\section{Resultados}

Todas las accesiones presentaron niveles detectables de los tres parámetros evaluados. Al comparar los contenidos globales extraídos de forma general para cada uno de los parámetros evaluados por municipios, se observó que solo en el caso de CFT hubo una ligera diferencia en contenidos, en el caso de la accesión del municipio de Llera. Por otra parte, la actividad contra ABTS y DPPH no presentó diferencias significativas entre las accesiones (Cuadro 2). La detección de CFT y fitoquímicos con propiedades antioxidantes en las diferentes accesiones de verdolaga, mostraron diferencias significativas en los tres parámetros evaluados $(p \leq 0.05)$ en el caso del solvente utilizado en el proceso de extracción, lo que permite reconocer la obtención diferencial de los componentes fitoquímicos de acuerdo con su polaridad (Cuadro 3).

Cuadro 2. Comparación de medias de los contenidos globales de compuestos fenólicos totales CFT, actividad colectora de radicales libres contra ABTS y DPPH en las diferentes accesiones.

\begin{tabular}{cccc}
\hline Municipio & CFT $\left(\mathrm{mEAG} \mathrm{g}^{-1}\right.$ PS $)$ & ABTS $\left(\mathrm{mM} \mathrm{ET} \mathrm{g}^{-1}\right.$ PS $)$ & DPPH $\left(\mathrm{mM} \mathrm{ET} \mathrm{g}^{-1}\right.$ PS $)$ \\
\hline $\mathrm{AB}$ & $3.9797 \pm 1.48 \mathrm{a}$ & $1.9642 \pm 1.01 \mathrm{a}$ & $1.4643 \pm 0.41 \mathrm{a}$ \\
$\mathrm{JN}$ & $3.88841 \pm 0.3 \mathrm{a}$ & $2.268 \pm 0.71 \mathrm{a}$ & $1.5164 \pm 0.28 \mathrm{a}$ \\
$\mathrm{LL}$ & $2.4383 \pm 0.81 \mathrm{ab}$ & $2.3492 \pm 0.55 \mathrm{a}$ & $1.4852 \pm 0.25 \mathrm{a}$ \\
$\mathrm{PA}$ & $3.1377 \pm 1.95 \mathrm{a}$ & $1.9025 \pm 1.07 \mathrm{a}$ & $1.4299 \pm 0.35 \mathrm{a}$ \\
$\mathrm{SN}$ & $3.758 \pm 0.61 \mathrm{a}$ & $2.0682 \pm 0.73 \mathrm{a}$ & $1.4615 \pm 0.32 \mathrm{a}$ \\
\hline
\end{tabular}

Letras distintas en la misma columna indican diferencias estadísticas significativas entre las accesiones $(p \leq 0.05)$.

El solvente utilizado influyó de manera importante en los parámetros analizados. Cuando se realizó la comparación general de los niveles de los parámetros evaluados con relación al solvente, fue notoria la diferencia de los solventes orgánicos con respecto a los niveles extraídos con el agua. El agua fue el solvente que permitió extraer la mayor cantidad de compuestos. Para los tres parámetros, la acetona y el etanol extrajeron niveles estadísticamente similares (Cuadro 3).

Cuadro 3. Comparación de medias de los contenidos globales de compuestos fenólicos totales, actividad colectora de radicales libres contra ABTS y DPPH extraídas con diferentes solventes.

\begin{tabular}{cccc}
\hline Solvente & CFT $\left(\mathrm{mM} \mathrm{ET} \mathrm{g}^{-1} \mathrm{PS}\right)$ & ABTS $\left(\mathrm{mM} \mathrm{ET} \mathrm{g}^{-1}\right.$ PS $)$ & DPPH $\left(\mathrm{mM} \mathrm{ET} \mathrm{g}^{-1} \mathrm{PS}\right)$ \\
\hline Acetona & $2.9114 \pm 0.75 \mathrm{~b}$ & $1.4561 \pm 0.57 \mathrm{~b}$ & $1.2618 \pm 0.13 \mathrm{~b}$ \\
Agua & $4.5781 \pm 0.97 \mathrm{a}$ & $3.1385 \pm 0.1 \mathrm{a}$ & $1.894 \pm 0.09 \mathrm{a}$ \\
Etanol & $2.7769 \pm 1.15 \mathrm{~b}$ & $1.686 \pm 0.22 \mathrm{~b}$ & $1.2341 \pm 0.08 \mathrm{~b}$ \\
\hline
\end{tabular}

Letras distintas en la misma columna indican diferencias estadísticas significativas entre las accesiones $(p \leq 0.05)$.

Los contenidos de CFT en cada accesión con respecto al solvente acetona mostraron que los niveles más altos fueron extraídos en las accesiones $\mathrm{AB}$ y SN, mientras las más bajas con este solvente fueron LL y PA. En el caso del contenido más alto registrado con el solvente agua fueron las accesiones $\mathrm{AB}$ y PA, mientras que, la más baja fue LL. El contenido más alto de CFT usando etanol para la extracción fue con la accesión JN y la más baja para la accesión PA (Cuadro 4). Se observaron perfiles heterogéneos con respecto a los niveles de CFT dependiendo del solvente, la mayoría de las accesiones mostraron los mayores niveles con el agua, excepto JN. 
Cuadro 4. Comparación de los contenidos de los compuestos fenólicos totales (CFT) (mEAG $\mathbf{g}^{-1}$ PS) cuantificados en las muestras de $\boldsymbol{P}$. oleracea extraídas con diferentes solventes.

\begin{tabular}{cccc}
\hline Accesión & Acetona & Agua & Etanol \\
\hline AB & $3.2678 \pm 0.11 \mathrm{~d}$ & $5.8938 \pm 0.03 \mathrm{a}$ & $2.6984 \pm 0.61 \mathrm{f}$ \\
JN & $3.0226 \pm 0.09 \mathrm{e}$ & $3.7228 \pm 0.22 \mathrm{c}$ & $4.2194 \pm 0.16 \mathrm{~b}$ \\
LL & $2.0944 \pm 0.48 \mathrm{~g}$ & $3.4291 \pm 0.4 \mathrm{~cd}$ & $1.7914 \pm 0.2 \mathrm{gh}$ \\
PA & $1.9959 \pm 0.45 \mathrm{~g}$ & $5.3742 \pm 0.11 \mathrm{a}$ & $1.2409 \pm 0.45 \mathrm{~h}$ \\
SN & $3.2104 \pm 0.29 \mathrm{~d}$ & $4.4707 \pm 0.21 \mathrm{~b}$ & $3.5929 \pm 0.37 \mathrm{~cd}$ \\
\hline
\end{tabular}

Letras distintas en la misma columna indican diferencias estadísticas significativas entre las accesiones $(p \leq 0.05)$.

La capacidad colectora contra el radical libre ABTS más altas se obtuvieron usando como solvente el agua, siendo las accesiones $\mathrm{AB}$ y JN las de mayor contenido. Por otro lado, el valor más alto alcanzado durante la extracción con acetona fue el de la accesión LL, mayor que el contenido obtenido en los extractos con etanol y por el contrario, el valor más bajo contra ABTS se registró en la accesión PA (Cuadro 5).

Cuadro 5. Comparación de los contenidos actividad colectora de radicales libres contra ABTS $\left(\mathrm{mM} \mathrm{ET}^{-1} \mathrm{PS}\right)$ cuantificados en las muestras de verdolaga (P. oleracea) extraídas con diferentes solventes.

\begin{tabular}{cccc}
\hline Accesión & Acetona & Agua & Etanol \\
\hline $\mathrm{AB}$ & $0.9539 \pm 0.28 \mathrm{~h}$ & $3.27 \pm 0.06 \mathrm{a}$ & $1.5565 \pm 0.09 \mathrm{fg}$ \\
$\mathrm{JN}$ & $1.6494 \pm 0.23 \mathrm{f}$ & $3.2226 \pm 0.04 \mathrm{a}$ & $1.9321 \pm 0.04 \mathrm{~d}$ \\
$\mathrm{LL}$ & $2.2076 \pm 0.06 \mathrm{c}$ & $3.069 \pm 0.01 \mathrm{~b}$ & $1.7711 \pm 0.05 \mathrm{de}$ \\
$\mathrm{PA}$ & $0.8135 \pm 0.02 \mathrm{i}$ & $3.099 \pm 0.07 \mathrm{~b}$ & $1.2974 \pm 0.12 \mathrm{~g}$ \\
$\mathrm{SN}$ & $1.3862 \pm 0.28 \mathrm{~g}$ & $3.032 \pm 0.07 \mathrm{~b}$ & $1.7863 \pm 0.06 \mathrm{de}$ \\
\hline
\end{tabular}

Letras distintas en la misma columna indican diferencias estadísticas significativas entre las accesiones $(p \leq 0.05)$.

Los niveles de capacidad colectora contra el radical DPPH mostraron que la accesión LL fue la de mayor contenido cuando se utilizó acetona como solvente y la más baja fue PA. En el caso del agua como solvente, se observó que la accesión $\mathrm{AB}$ fue la más alta y la accesión LL la más baja. En el caso del etanol, la accesión JN fue la que presentó el nivel mayor de capacidad colectora en tanto que PA tuvo los niveles menores (Cuadro 6).

Cuadro 6. Comparación de los contenidos actividad colectora de radicales libres contra DPPH (mM ET $\left.\mathrm{g}^{-1} \mathrm{PS}\right)$ cuantificados en las muestras de verdolaga (P. oleracea) extraídas con diferentes solventes en los diferentes municipios de estudio.

\begin{tabular}{cccc}
\hline Accesión & Acetona & Agua & Etanol \\
\hline $\mathrm{AB}$ & $1.1457 \pm 0.04 \mathrm{~g}$ & $2.0204 \pm 0.05 \mathrm{a}$ & $1.1915 \pm 0.02 \mathrm{fg}$ \\
$\mathrm{JN}$ & $1.2803 \pm 0.05 \mathrm{e}$ & $1.9038 \pm 0.04 \mathrm{~b}$ & $1.3651 \pm 0.03 \mathrm{de}$ \\
$\mathrm{LL}$ & $1.4526 \pm 0.09 \mathrm{~d}$ & $1.7963 \pm 0.04 \mathrm{c}$ & $1.2068 \pm 0.06 \mathrm{fg}$ \\
$\mathrm{PA}$ & $1.1174 \pm 0.08 \mathrm{gh}$ & $1.8411 \pm 0.08 \mathrm{bc}$ & $1.169 \pm 0.06 \mathrm{~g}$ \\
$\mathrm{SN}$ & $1.2521 \pm 0.08 \mathrm{e}$ & $1.9087 \pm 0.04 \mathrm{~b}$ & $1.2238 \pm 0.04 \mathrm{f}$ \\
\hline
\end{tabular}

Letras distintas en la misma columna indican diferencias estadísticas significativas entre las accesiones $(p \leq 0.05)$. 
Lo anterior, muestra que existe una heterogeneidad importante con relación a cada accesión y respecto a la naturaleza polar de los componentes fenólicos y las capacidades colectoras de radicales libres en todas las accesiones. El agua resultó como el solvente que mayor capacidad de extracción tiene frente a solventes orgánicos evaluados. Solo la accesión JN tuvo los niveles de CFT más altos con etanol.

\section{Discusiones}

Los niveles de CFT y la capacidad colectora de radicales libres en las accesiones de $P$. oleracea evaluadas, permiten observar una marcada heterogeneidad cuando se comparan bajo una misma condición de extracción. Aunque la procedencia de las muestras corresponde con zonas de crecimiento diferentes y distantes, aún restan estudios profundos para determinar el efecto de las condiciones ambientales en los niveles de cada parámetro, aunque la literatura señala que estas podrían influir en el balance de antioxidantes y compuestos fenólicos, considerándose como respuestas adaptativas frente a situaciones de estrés (Gharibi et al., 2016; Kaur et al., 2017).

Los niveles de CFT y antioxidantes están directamente relacionados con procesos de señalización en las plantas, así como defensa contra herbívoros, protección contra el estrés lumínico, tolerancia contra metales pesados y para resguardar el balance redox de las plantas; por lo cual, sus niveles dependerán de las condiciones donde se desarrollen las plantas. Los contenidos detectados en el presente estudio son similares a los reportados por investigaciones anteriores (Santiago-Saenz et al., 2018; Sicari et al., 2018; Habibian et al., 2020) aunque, la identidad química de los agentes responsables de los efectos antioxidantes es desconocida en las accesiones de Tamaulipas.

La naturaleza del solvente empleado durante la extracción permitió hacer una exploración más completa de los contenidos de compuestos fenólicos y fitoquímicos con actividad colectora de radicales libres en las accesiones silvestres ya que, se ha observado que el solvente tiene una fuerte influencia en la diversidad y concentraciones obtenidas de fitoquímicos con actividades antioxidantes, que atribuye al efecto de la polaridad (Felhi et al., 2017; Thouri et al., 2017). Esto coincide con los resultados que muestran que la mayoría de las accesiones de $P$. oleracea tuvieron contenidos superiores en extractos acuosos y algunas de forma diferencial entre acetona y etanol.

Esta influencia del solvente sobre los niveles y naturaleza de los fitoquímicos extraídos se ha reportado anteriormente en $P$. oleracea (Habibian et al., 2020), donde además se señala que los contenidos de CFT y los niveles de capacidad colectora contra ABTS y DPPH fueron similares entre extractos etanólicos y acuosos, tal como ocurrió para la mayoría de las accesiones en este estudio. Se ha reportado que los solventes con mayor polaridad permiten la extracción de mayores contenidos de CFT y capacidad colectora de radicales libres, esto explica porque el agua fue más eficiente para extraer estos metabolitos de P. oleracea (Almulaiky et al., 2020).

La caracterización de variedades silvestres de verdolaga y el conocimiento de las condiciones óptimas de crecimiento, son aspectos clave durante los procesos de manejo. Debido a que las formas silvestres, constituyen reservorios de información genética y respuestas fisiológicas que pudieran en algún momento, ser transferidas de los parientes silvestres a formas cultivadas. Ya sea mediante mejoramiento genético o modulada mediante la modificación de los ambientes, para que se favorezca la acumulación o disminución de un fitoquímico determinado (Van Treuren et al., 2018; Araghi et al., 2019). 
La variación en aspectos fisiológicos primarios y secundarios es un fenómeno ya reportado para $P$. oleracea, donde accesiones silvestres responden de forma diferencial a la regeneración, evapotranspiración, fotosíntesis y contenido de clorofila (Alam et al., 2014). También, se ha señalado que existe una alta variabilidad en la acumulación de compuestos de naturaleza fenólica entre variedades de $P$. oleracea, algo que coincide con lo observado en las accesiones de este trabajo. Esta variación debe ser considerada como una parte importante para el manejo de $P$. oleracea, debido a que pudiera comprometer los contenidos de compuestos de importancia alimenticia y los compuestos bioactivos (Sdouga et al., 2020).

Los contenidos de CFT observados en las accesiones de P. oleracea detectados en este trabajo son menores a los valores reportados en estudios anteriores, que varían entre 5.57 y $21.61 \mathrm{mg}$ EAG $\mathrm{g}^{-1} \mathrm{PS}$, lo cual pudiera estar asociado al tipo de extracción empleada y la polaridad del solvente usado (Gallo et al., 2017; Gatea; et al., 2017). Respecto a la influencia de la polaridad en los niveles extraídos, a partir de P. oleracea se ha reportado que las mezclas hidroalcohólicas con contenidos de 30 y $50 \%$ tienen mejores rendimientos que el agua sola o el etanol absoluto (Gallo et al., 2017), lo cual, abre la posibilidad de que en los tejidos de las accesiones de Tamaulipas pudieran existir valores que no se detectaron por la influencia del solvente.

Respecto a los contenidos de capacidad colectora contra el radical ABTS, se detectaron niveles mayores a los reportados en estudios previos, donde se señalan contenidos entre 4.71 a $74.6 \mu \mathrm{M}$ de ET $\mathrm{g}^{-1} \mathrm{PS}$, en los cuales también se mencionó la influencia de las condiciones de crecimiento y la naturaleza del solvente utilizado, destacándose que las fases de etanol al 30 y $50 \%$ resultaron más eficientes en la extracción (Gatea et al., 2017; Santiago-Saenz et al., 2018).

En el caso de la capacidad colectora contra el radical DPPH los valores registrados en este trabajo fueron mayores a lo reportado por Santiago-Saenz et al. (2018), al comparar los contenidos de $P$. oleracea con el quelite cenizo (Chenopodium berlandieri L.) y el quintonil (Amaranthus hybridus L.), la verdolaga y el quelite cenizo tuvieron los contenidos más altos de CFT y capacidad colectora contra ABTS. P. oleracea, presentó mayor capacidad colectora contra el DPPH, mientras que el quintonil, fue el más bajo respecto a los tres parámetros señalados (Santiago-Saenz et al., 2018). Esto señala a la $P$. oleracea como una fuente importante de compuestos con capacidades antioxidantes potenciales que pudieran darle un valor agregado a esta planta como material alimenticio.

Al comparar los contenidos de los parámetros evaluados en las accesiones de P. oleracea de Tamaulipas, se observó que todas tuvieron contenidos de CFT menores a los de diversas plantas usadas como condimentos; no obstante, respecto a los niveles de capacidad colectora contra ABTS se encuentran dentro de los rangos reportados para 39 especias usadas como condimentos.

En el caso de los valores registrados para la capacidad colectora contra el DPPH de las accesiones silvestres, esta se encuentra en rangos similares a los contenidos reportados para especies de uso culinario y medicinal (Chan et al., 2016; Assefa et al., 2018). Los resultados del presente estudio indican el potencial alimenticio y funcional de la verdolaga silvestre de Tamaulipas, ya que muestra contenidos de compuestos con capacidad colectora de radicales libres equivalentes a los de varias plantas comestibles o de uso medicinal, lo cual refuerza su potencial para consumo. 
Es importante remarcar que la variación encontrada en las accesiones pudiera ser atribuida a las condiciones climáticas y de crecimiento; no obstante, hacen falta estudios más profundos para designar que parámetros tienen más influencia en estas variaciones. También hay que considerar que, al ser accesiones silvestres, deben ser evaluadas para determinar parámetros de importancia en la nutrición como parte de los conocimientos necesarios para el aprovechamiento de esta especie con fines alimenticios y nutraceúticos o para planes de manejo de la especie.

\section{Conclusiones}

Las accesiones silvestres de $P$. oleracea en Tamaulipas presentaron una variación a niveles de compuestos fenólicos y la capacidad colectora de radicales libres, en estos, el tipo de solvente tuvo una influencia en su extracción. Estos parámetros son importantes y deben ser considerados para el aprovechamiento de este recurso natural y el desarrollo de productos para el consumo humano.

\section{Agradecimientos}

Al CONACYT por la beca de doctorado para el primer autor (número de beca: 726281) al proyecto 'Desarrollo y trasferencia de tecnología para la producción orgánica de verdolaga autóctona (Portulaca oleracea L.) en el noreste de México, como una alternativa para disminuir la desnutrición, fomentando el uso y consumo regional' 2017-5 apoyado por FONSEC-SAGARPACONACYT (SA-3888). Gracias a la Dra. Reyna Ivonne Torres Acosta por el apoyo durante el trabajo de campo.

\section{Literatura citada}

Alam, M.; Juraimi, A. S.; Rafii, M. Y.; Abdul, A.; Aslani, F.; Hasan, M. M.; Zainudin, M. A. and Uddin, M. 2014. Evaluation of antioxidant compounds, antioxidant activities, and mineral composition of 13 collected purslane (Portulaca oleracea L.) accessions. BioMed Res. Int. 2014(1):1-6. https://doi.org/10.1155/2014/951019.

Allahmoradi, E.; Taghiloo, S.; Omrani-Nava, V.; Shobeiri, S. S.; Tehrani, M.; Ebrahimzadeh, M. A. and Asgarian-Omran, H. 2018. Antiinflammatory effects of the Portulaca oleracea hydroalcholic extract on human peripheral blood mononuclear cells. Med. J. Islam Repub. Iran. 32(1):1-6.

Almulaiky, Y. Q.; Aldhahri, M.; Al-abbasi, F. A.; Al-Harbi, S. A. and Shiboob, M. H. 2020. In Vitro Assessment of Antioxidant Enzymes, Phenolic Contents and Antioxidant Capacity of the Verdolaga (Portulacaceae). Int. J. Nutr. 4(4):36-47. https://doi.org/10.14302/ issn.2379-7835.ijn-19-3144.

Alu'datt, M. H.; Rababah, T.; Alhamad, M. N.; Al-Tawaha, A.; Al-Tawaha, A. R.; Gammoh, S.; Ereifeja, K. I.; Al-Karakic, G.; Hamashae, F.; Tranchantg, C. C. and Kubow, S. 2019. Herbal yield, nutritive composition, phenolic contents and antioxidant activity of purslane (Portulaca oleracea L.) grown in different soilless media in a closed system. Ind. Crop Prod. 141:11746. https://doi.org/10.1016/j.indcrop.2019.111746.

Araghi, A. M.; Nemati, H.; Azizi, M.; Moshtaghi, N.; Shoor, M. and Hadian, J. 2019. Assessment of phytochemical and agro-morphological variability among different wild accessions of Mentha longifolia L. cultivated in field condition. Ind. Crop. Prod. 140:111698. https://doi.org/10.1016/j.indcrop.2019.111698. 
Assefa, A. D.; Keum, Y. S. and Saini, R. K. 2018. A comprehensive study of polyphenols contents and antioxidant potential of 39 widely used spices and food condiments. J. Food Meas. Charact. 12(3):1548-1555. https://doi.org/10.1007/s11694-018-9770-z.

Bartolini, S.; Leccese, A.; Remorini, D.; Iacona, C. and Viti, R. 2018. Quality and antioxidant traits of organic apricots (Prunus armeniaca L.) at harvest and after storage. Eur. J. Hortic. Sci. 83(1):12-17. https://doi.org/10.17660/eJHS.2018/83.1.2.

Bautista, I.; Boscaiu, M.; Lidón, A.; Llinares, J. V.; Lull, C.; Donat, M. P.; Mayoral, O. and Vicente, O. 2016. Environmentally induced changes in antioxidant phenolic compounds levels in wild plants. Acta Physiol. Plant. 38(1):1-15. https://doi.org/10.1007/s11738-0152025-2.

Brand-Williams, W.; Cuvelier, M. E. and Berset, C. L. W. T. 1995. Use of a free radical method to evaluate antioxidant activity. LWT - Food Sci. Technol. 28(1):25-30. https://doi.org/ 10.1016/ S0023-6438(95)80008-5.

Capecka, E.; Mareczek, A. and Leja, M. 2005. Antioxidant activity of fresh and dry herbs of some Lamiaceae species. Food Chem. 93(2):223-226. https://doi.org/10.1016/j.foodchem. 2004.09.020.

Caverzan, A.; Casassola, A. and Brammer, S. P. 2016. Antioxidant responses of wheat plants under stress. Genet. Mol. Biol. 39(1):1-6. https://doi.org/10.1590/1678-4685-GMB-2015-0109.

Chan, C. L.; Gan, R. Y. and Corke, H. 2016. The phenolic composition and antioxidant capacity of soluble and bound extracts in selected dietary spices and medicinal herbs. Int. J. Food Sci. Tech. 51(3):565-573. https://doi.org/10.1111/ijfs.13024.

Cobaleda-Velasco, M.; Alanis-Bañuelos, R. E.; Almaraz-Abarca, N.; Rojas-López, M.; GonzálezValdez, L. S.; Ávila-Reyes J. A. and Rodrigo S. 2017. Phenolic profiles and antioxidant properties of Physalis angulata L. as quality indicators. J. Pharm. Pharmacogn. Res. 5(2):114-128.

Felhi, S.; Daoud, A.; Hajlaoui, H.; Mnafgui, K.; Gharsallah, N. and Kadri, A. 2017. Solvent extraction effects on phytochemical constituents profiles, antioxidant and antimicrobial activities and functional group analysis of Ecballium elaterium seeds and peels fruits. Food Sci. Technol. 37(3):483-492. http://dx.doi.org/10.1590/1678-457x.23516.

Frontela, C.; Canali, R. y Virgili, F. 2010. Empleo de compuestos fenólicos en la dieta para modular la respuesta inflamatoria intestinal. Gastroenterol Hepatol. 33(4):307-312. https://doi.org/10.1016/j.gastrohep.2009.09.006.

Gallo, M.; Conte, E. and Naviglio, D. 2017. Analysis and comparison of the antioxidant component of Portulaca oleracea leaves obtained by different solid-liquid extraction techniques. Antioxidants. 6(3):64. https://doi.org/10.3390/antiox6030064.

García, E. 2004. Modificaciones al sistema de clasificación climática de Köppen. $5^{\text {a }}$. (Ed.). Instituto de Geografía-Universidad Nacional Autónoma de México (UNAM). DF, México. 92 p.

Gatea, F.; Teodor, E. D.; Seciu, A. M.; Nagodă, E. and Radu, G. L. 2017. Chemical constituents and bioactive potential of Portulaca pilosa L vs. Portulaca oleracea L. Med. Chem. Res. 26(7):1516-1527. https://doi.org/10.1007/s00044-017-1862-5.

Gharibi, S.; Tabatabaei, B. E. S.; Saeidi, G. and Goli, S. A. H. 2016. Effect of drought stress on total phenolic, lipid peroxidation, and antioxidant activity of Achillea species. Appl. Biochem. Biotechnol. 178(4):796-809. https://doi.org/10.1007/s12010-015-1909-3.

Gutiérrez, Á.; Ledesma, L.; García, I. y Grajales, O. 2007. Capacidad antioxidante total en alimentos convencionales y regionales de Chiapas, México. Rev. Cub. Salud Pública. 33(1):1-7. 
Habibian, M.; Sadeghi, G. and Karimi, A. 2020. Phytochemicals and Antioxidant Properties of Solvent Extracts from Purslane (Portulaca oleracea L.): a preliminary study. Food Sci. Eng. 1(1):1-12. https://doi.org/10.37256/fse.11202046.

Hammer, O.; Harper, D. A. T. and Ryan, P. D. 2001. PAST: paleontological statistics software package for education and data analysis. Paleontol. Electron. 4(1):1-9.

Kaur, D.; Grewal, S. K.; Kaur, J. and Singh, S. 2017. Free radical scavenging activities can mitigate the effect of water stress in chickpea. Crop Pasture Sci. 68(6):544-554. https://doi.org/10.1071/CP17022.

Khodadadi, H.; Pakdel, R.; Khazaei, M.; Niazmand, S.; Bavarsad, K. and Hadjzadeh, M. 2018. A comparison of the effects of Portulaca oleracea seeds hydro-alcoholic extract and Vitamin $\mathrm{C}$ on biochemical, hemodynamic and functional parameters in cardiac tissue of rats with subclinical hyperthyroidism. Avicenna J. Phytomed. 8(2):161-169.

Mocan, A.; Zengin, G.; Simirgiotis, M.; Schafberg, M.; Mollica, A.; Vodnar, D. C.; Crişan, G. and Rohn, S. 2017. Functional constituents of wild and cultivated Goji (L. barbarum L.) leaves: phytochemical characterization, biological profile, and computational studies. J. Enzyme Inhib. Med. Chem. 32(1):153-168. https://doi.org/10.1080/14756366.2016.1243535.

Nemzer, B.; Al-Taher, F. and Abshiru, N. 2020. Phytochemical composition and nutritional value of different plant parts in two cultivated and wild purslane (Portulaca oleracea L.) genotypes. Food Chem. 320(1):126621. https://doi.org/10.1016/j.foodchem.2020. 126621.

Ninfali, P.; Mea, G.; Giorgini, S.; Rocchi, M. and Bacchiocca, M. 2005. Antioxidant capacity of vegetables, spices and dressings relevant to nutrition. Br. J. Nutr. 93(2):257-266. https://doi.org/10.1079/BJN20041327.

Oliveira, I.; Valentao, P.; Lopes, R.; Andrade, P. B.; Bento, A. and Pereira, J. A. 2009. Phytochemical characterization and radical scanvenging activity of Portulaca oleracea L. leaves and Stems. Microchem. 92(2):129-134. https://doi.org/10.1016/j.microc.2009. 02.006.

Qasim, M.; Abideen, Z.; Adnan, M. Y.; Gulzar, S.; Gul, B.; Rasheed, M. and Khan, M. A. 2017. Antioxidant properties, phenolic composition, bioactive compounds and nutritive value of medicinal halophytes commonly used as herbal teas. S. African J. Bot. 110(1):240-250. https://doi.org/10.1016/j.sajb.2016.10.005.

Ramadan, B. K.; Schaalan, M. F. and Tolba, A. M. 2017. Hypoglycemic and pancreatic protective effects of Portulaca oleracea extract in alloxan induced diabetic rats. BMC Complement. Altern. Med. 17(1):37. https://doi.org/10.1186/s12906-016-1530-1.

Re, R.; Pellegrini, N.; Proteggente, A.; Pannala, A.; Yang, M. and Rice-Evans, C. 1999. Antioxidant activity applying an improved ABTS radical cation decolorization assay. Free Radic. Biol. Med. 26(9-10):1231-1237. https://doi.org/10.1016/S0891-5849(98)00315-3.

Román, N. R.; Del Rosario, M.; Castillo, A. M.; Sahagún, J. y Jiménez, M. A. 2018. Características nutricionales y nutracéuticas de hortalizas de uso ancestral en México. Rev. Fitotec. Mex. 41(3):245-253. https://doi.org/10.35196/rfm.2018.3.245-253.

Santiago-Saenz, Y. O.; Hernández-Fuentes, A. D.; Monroy-Torres, R.; Cariño-Cortés, R. and Jiménez-Alvarado, R. 2018. Physicochemical, nutritional and antioxidant characterization of three vegetables (Amaranthus hybridus L., Chenopodium berlandieri L., Portulaca oleracea L.) as potential sources of phytochemicals and bioactive compounds. J. Food Meas. Charact. 12(4):2855-2864. https://doi.org/10.1007/s11694-018-9900-7. 
Sauceda, A. E. Q.; Palafox, H.; Sánchez, R. M. R. y Aguilar, G. A. G. 2011. Interacción de compuestos fenólicos y fibra dietaria: capacidad antioxidante y biodisponibilidad. Biotecnia. 13(3):3-11. https://doi.org/10.18633/bt.v13i3.91.

Sdouga, D.; Branca, F.; Kabtni, S.; Trifi-Farah, N. and Marghali, S. 2020. Polyphenol variability of Italian and Tunisian populations of Portulaca oleracea L. Acta Hortic. 1267(1):115-118. https://doi.org/10.17660/ActaHortic.2020.1267.18.

Sicari, V.; Loizzo, M. R.; Tundis, R.; Mincione, A. and Pellicano, T. M. 2018. Portulaca oleracea L. (Purslane) extracts display antioxidant and hypoglycaemic effects. J. Appl. Bot. Food Qual. 91(1):39-46. https://doi.org/10.5073/JABFQ.2018.091.006.

Singleton, V. L.; Orthofer, R. and Lamuela-Raventós, R. M. 1999. Analysis of total phenols and other oxidation substrates and antioxidants by means of Folin-Ciocalteu reagent. Methods Enzymol. 299(1):152-178. Academic press. https://doi.org/10.1016/S00766879(99)99017-1.

Thouri, A.; Chahdoura, H.; El Arem, A.; Hichri, A. O.; Hassin, R. B. and Achour, L. 2017. Effect of solvents extraction on phytochemical components and biological activities of Tunisian date seeds (var. Korkobbi and Arechti). BMC Complement. Altern. Med. 17(1):248. https://doi.org/10.1186/s12906-017-1751-y.

Top, O.; Bar, C.; Ökmen, B.; Özer, D. Y.; Rusçuklu, D.; Tamer, N.; Frary, A. and Doğanlar, S. 2014. Exploration of three solanum species for improvement of antioxidant traits in tomato. HortScience. 49(8):1003-1009. https://doi.org/10.21273/HORTSCI.49.8.1003.

Urbizu-González, A. L.; Castillo-Ruiz, O.; Martínez-Ávila, G. C. G. and Torres-Castillo, J. A. 2017. Natural variability of essential oil and antioxidants in the medicinal plant Turnera diffusa. Asian Pac. J. Trop. Med. 10(2):121-125. https://doi.org/10.1016/j.apjtm.2017. 01.013 .

Van Treuren, R.; Van Eekelen, H. D.; Wehrens, R. and de Vos, R. C. 2018. Metabolite variation in the lettuce gene pool: towards healthier crop varieties and food. Metabolomics. 14(11):146. https://doi.org/10.1007/s11306-018-1443-8. 